\title{
Single incision laparoscopic splenectomy, technical aspects and feasibility considerations
}

\author{
Francesco Cabras ${ }^{1}$, Lazzara Fabrizio ${ }^{1}$, Umberto Bracale ${ }^{1,2}$, Jacopo Andreuccetti ${ }^{1}$, Giusto Pignata ${ }^{1}$ \\ ${ }^{1}$ General and Mini-Invasive Surgery, "San Camillo" Hospital, Trento, Italy \\ ${ }^{2}$ Department of Surgery, University of Naples “Federico II”, Naples, Italy
}

Videosurgery Miniinv 2014; 9 (4): 632-633

DOI: $10.5114 /$ wiitm.2014.44137

\begin{abstract}
Minimally invasive techniques have been introduced to reduce morbidity related to standard laparoscopic procedures. One such approach is laparoendoscopic single-site surgery. The aim of the study was to present our initial clinical experience of using this technique for elective splenectomy. We carried out single access laparoscopic splenectomy (SALS) for an $8 \mathrm{~cm}$ cystic lesion of the spleen, involving the hilum, on a 38-year-old woman. The procedure was performed with a single-port device (4-channel) via a $2.5-\mathrm{cm}$ umbilical incision. A flexible 5-mm optic and straight laparoscopic instruments were used. The operative time was $75 \mathrm{~min}$. There was no blood loss. No complications were observed. The postoperative period was uneventful. Although substantial development of the instruments and skills is needed, this SALS technique appears to be feasible and safe. Nevertheless, further experience and observations are necessary.
\end{abstract}

Key words: laparoscopy, laparoscopic splenectomy, single incision laparoscopic surgery, spleen, minimally invasive splenic surgery.

\section{Introduction}

The laparoscopic approach has gained acceptance in many surgical fields [1]. Splenectomy is a very common surgical procedure but with varied indications. Depending on the surgical indications, the operative steps, difficulty of the surgical procedure, and postoperative outcomes could differ. Laparoscopic splenectomy was first described by Rhodes et al. [2]; since then, it has been widely used in clinical practice. It is demonstrated that laparoscopic splenectomy (LS) is associated with more favourable outcomes than open surgery [3]. Single access laparoscopic splenectomy (SALS) could be a step forward in the way to invasiveness reduction. After the introduction in our operative practice of some SALS procedures $[4,5]$, we tried to reproduce the same surgi- cal principles of standard LS through a single access approach. We report a video about a single incision laparoscopic splenectomy showing some technical aspects and feasibility considerations.

The aim of this report was to assess the feasibility and safety of SALS with laparoendoscopic single-site surgery (LESS) for a supposed benign lesion.

\section{Case report}

The video shows a case of a 38-year-old woman complaining of left flank pain. Computed tomography (CT) scan showed a voluminous cystic lesion ( $8 \mathrm{~cm}$ in diameter) of the lower pole of the spleen, involving the hilum. In consideration of the case history, and particularly the involvement of the hilum, the decision to perform a total splenectomy 
was taken. After meningococcal, pneumococcal, and Haemophilus influenzae type B preoperative preparation, the splenectomy was carried out, in the right lateral position, first with a vascular approach and then with spleen lateral mobilization. The procedure was performed with a single-port device (4-channel) via a $2.5-\mathrm{cm}$ umbilical incision. A flexible 5-mm laparoscope (5 $\mathrm{mm}$ flexible handle laparoscope) and straight laparoscopic instruments were used.

The splenectomy was carried out in 75 min without intraoperative complications. The postoperative period was uneventful. Histology described a benign cystic lesion, confirming its proximity to the hilum. The patient has had the problem of pain solved with an aesthetic $2.5 \mathrm{~cm}$ umbilical scar.

\section{Discussion}

The introduction of laparoscopy in the early 1990s ushered in a new era in the surgical treatment of human diseases. Other less invasive procedures have been applied to splenic surgery since Barbaros and Dinccag first reported in 2009 [6]. Theoretically, the increase in the number of incisions and port sites may elevate the incidence of abdominal wall hernia and wound infection. The SALS could potentially minimize these complications.

In transumbilical SALS, the incision is hidden in the natural umbilical skin fold so that the postoperative scar is almost invisible. This surgical technique is therefore called "no scar surgery". It has advantages of less traumatic and better cosmetic outcomes than multi-port laparoscopy. However, the length of skin incision is partly determined by the size of a resected specimen, which is especially true in cases of splenic malignancies that require integral specimens for histologic analysis.

It is undeniable that SALS has several disadvantages compared with multiport laparoscopic surgery in terms of surgical instruments and techniques. Although SALS can be performed with a conventional rigid laparoscope and straight instruments, crowding over the access port or access site usually leads to clashing of surgical instruments. The parallel arrangement of instruments and the constant interference between the surgeon and the camera operator both add difficulty to the procedure. Furthermore, lack of tissue triangulation significantly increases the difficulty of splenic exposure and dissection.

On this point, a small advantage, in our case, was granted by the use of a flexible camera that allows the assistant not to interfere with the movements of the operator surgeon by staying at his side or behind.

\section{Conclusions}

Early experiences show that SALS is feasible and safe in experienced hands. However, as an emerging operation, publication bias is a factor that should be considered before we can draw an objective conclusion. Whether there are potential benefits associated with SALS over LS is yet to be proven objectively. Unfortunately, most publications regarding single-access splenectomy are case reports with small series. Future multicenter randomized controlled trials are needed to determine its merits and shortcomings when compared with LS and also to explore the possible long-term complications, such as incisional hernia [7], as well as the cost-effectiveness of SALS.

\section{References}

1. Bracale U, Pignata G, Lirici MM, et al. Guideline Committee of the Italian Society of Hospital Surgeons-ACOI and Italian HiTech Surgical Club-IHTSC. Laparoscopic gastrectomies for cancer: The ACOI-IHTSC national guidelines. Minim Invasive Ther Allied Technol 2012; 21: 313-9.

2. Rhodes M, Rudd M, O’Rourke N, et al. Laparoscopic splenectomy and and lymph node biopsy for hematologic disorders. Ann Surg 1995; 222: 43-6.

3. Musallam KM, Khalife M, Sfeir PM, et al. Postoperative outcomes after laparoscopic compared with open splenectomy. Ann Surg 2013; 257: 1116-23.

4. Bracale U, Lazzara F, Merola G, et al. Single access laparoscopic left hemicolectomy with or without inferior mesenteric artery preservation: our preliminary experience. Minerva Chir 2013; 68: 315-20.

5. Bracale U, Nastro P, Bramante S, Pignata G. Single incision laparoscopic anterior resection for cancer using a "QuadiPort access system". Acta Chir lugosl 2010; 57: 105-9.

6. Barbaros U, Dinccag A. Single incision laparoscopic splenectomy: the first two cases. J Gastrointest Surg 2009; 13: 1520-3.

7. Marks JM, Phillips MS, Tacchino R, et al. Single-incision laparoscopic cholecystectomy is associated with improved cosmesis scoring at the cost of significantly higher hernia rates: 1-year results of a prospective randomized, multicenter, single-blinded trial of traditional multiport laparoscopic cholecystectomy vs single-incision laparoscopic cholecystectomy. J Am Coll Surg 2013; 216: 1037-47.

Received: 15.02.2014, accepted: 5.03.2014. 